\title{
The 2D coefficient inverse problem of the ultrasound waves propagation
}

\author{
N. Novikov ${ }^{1,3 *}$, M. Shishlenin ${ }^{1,2,3}$ \\ ${ }^{1}$ Institute of Computational Mathematics and Mathematical Geophysics SB RAS, Novosibirsk, Russia \\ ${ }^{2}$ Sobolev Institute of Mathematics SB RAS, Novosibirsk, Russia \\ ${ }^{3}$ Novosibirsk State University, Novosibirsk, Russia \\ *e-mail:novikov-1989@yandex.ru
}

Key words: inverse problem, acoustic equations, gradient descent method

Motivation and Aim: Ultrasound imaging is intensively developing the past two decades. Various methods have been developed, including magnetic resonance, positron emission, optical tomography, etc. x-ray tomography has become the most popular among these methods both in medicine and in industrial diagnostics. However, the disadvantage of medical x-ray tomography devices is a high dose of radiation that prevents their application in the regular examination. The limitation of exposure of ionizing radiation has become a current trend in medicine, which makes the development of methods of ultrasonic tomography of paramount importance. One of the major problems of ultrasound tomography is the development of methods for solving inverse problems which are nonlinear.

Methods and Algorithms: We consider the direct and inverse problem for the hyperbolic system of equations, that describes the two dimensional acoustic wave propagation. The hyperbolic first-order system allows us to propose more realistic model from the physical point of view. On the other hand, we can apply optimization approach for recovering coefficients of such system, like the density of the medium, the speed of the wave propagation or the attenuation coefficient. We consider the numerical algorithm for solving direct problems, based on the S.K. Godunov scheme. In order to solve the inverse problem, we use optimization approach and propose gradient-based method to minimize the cost functional.

Results: We present the result of direct problem solution. We obtain the gradient of the functional for recovering the density and the acoustic wave's velocity in the medium. We use it to obtain the solution of inverse problem and study the behavior of the cost functional.

Acknowledgements: Supported by the RFBR 16-29-15120 and by MSC RK grant No. AP05134121.

\section{References}

1. Kabanikhin S.I., Shishlenin M.A. (2008) Quasi-solution in inverse coefficient problems. Journal of Inverse and Ill-posed Problems. 16(7):705-713.

2. Kulikov I.M., Novikov N.S., Shishlenin M.A. (2015) Mathematical modelling of ultrasound wave propagation in two dimensional medium: direct and inverse problem. Siberian Electronic Mathematical Reports 12:219-228.

3. Kabanikhin S.I., Novikov N.S., Oseledets I.V., Shishlenin M.A. (2015) Fast Toeplitz linear system inversion for solving two-dimensional acoustic inverse problem. Journal of Inverse and Ill-posed Problems. 23(6):687-700. 